\title{
Gut microbial characteristics of adult patients with allergy rhinitis
}

\author{
Libing Zhu ${ }^{1}$, Feng $X u^{2}$, Wenrong Wan ${ }^{2}$, Bin Yu ${ }^{3}$, Lin Tang ${ }^{3}$, Yimin Yang ${ }^{4}$, Yanling Du ${ }^{5}$, Zhangran Chen ${ }^{6,7,8^{*}}$ (D) \\ and Hongzhi $X u^{6,7,8^{*}}$
}

\begin{abstract}
Background: Although recent studies have indicated that intestinal microbiota dweller are involved in the pathogenesis of allergy rhinitis (AR), the influence of gut microbiota on AR adult has not been fully elucidated yet. Hence, we carried out this study to uncover the distinctive bacterial taxa that differentiate allergy rhinitis patients from healthy individuals. Feces samples from thirty three AR patients and thirty one healthy individuals were analyzed by $16 \mathrm{~S}$ rRNA gene sequencing.

Results: Results showed that the bacterial diversity in AR group was significantly higher than that of the non-AR group. Bacterial communities between AR and non-AR group were significantly differentiated as revealed by Principal coordinates analysis (PCOA) and the variation within non-AR were higher than that of the counterpart. Firmicutes, Fusobacteria, Actinobacteria, Cyanobacteria and Chloroflexi were the significantly differed phyla taxa and the top significantly distinguished bacterial genus included Prevotella_9, Phascolarctobacterium, Roseburia, Megamonas, Alistipes, Lachnoclostridium and Fusobacterium. The higher network complexity in AR group were dominated by taxa belonging to Firmicutes. The predicted function, alpha linolenic acid metabolism and bacterial invasion of epithelial cells pathway were higher in non-AR group while gonadotropin-releasing hormone $(\mathrm{GnRH})$ signaling pathway, Fc $\gamma-\mathrm{R}$ mediated phagocytosis and endocytosis were higher in AR patients. Although the bacterial diversity between moderate and severe AR patients showed no significant difference, the significant correlation between featured genus and total nasal symptom score or rhinoconjunctivitis quality of life questionnaire, such as Butyricicoccus and Eisenbergiella, revealed the potential to intervene the AR status by means of gut microbiota.
\end{abstract}

Conclusions: In conclusion, patients with allergy rhinitis had distinguished gut microbiota characteritics in comparison with healthy controls. The results suggest that gut microbiota might play crucial roles in influencing the course and different symptoms of AR.

Trial registration ChiCTR, ChiCTR1900028613. Registered 29 December 2019, https://www.chictr.org.cn/showproj. aspx?proj=47650.

Keywords: Allergy rhinitis, Gut dysbiosis, Total nasal symptom score, Functional pathway

\footnotetext{
*Correspondence: zhangran22105@xmu.edu.cn; xuhongzhi@xmu.edu.cn

${ }^{6}$ Department of Digestive Diseases, School of Medicine, Xiamen

University, Xiamen, China

Full list of author information is available at the end of the article

The original version of this article was revised: Following publication of the original article [1], the authors identified an error in the author names of Hongzhi $X u$ and Yimin Yang. The incorrect author name is: Hongzhu Xu and Yiming Yang. The correct author name is: Hongzhi Xu and Yimin Yang. The author group has been updated above and the original article [1] has been corrected. The correct caption for the figure 1 has been included in this correction, and the original article has been corrected.
}

\begin{abstract}
Background
Allergic rhinitis (AR) is one of the most globally common diseases and usually persists throughout life $[1,2]$. AR is defined as immunoglobulin E (IgE)-mediated noninfective inflammatory disease of the nasal mucosa after allergen exposure and a variety of immune active cells and cytokines are involved [3]. The classic symptoms of AR include nasal congestion, nasal itching, rhinorrhea and
\end{abstract}


sneezing $[4,5]$. Conservatively, more than 500 million people suffer from allergic rhinitis around the world [1]. AR impose a significantly adverse effect on the quality of life [1] and increase a risk for bronchial asthma, rhinosinusitis, nasal polyps, otitis media and allergic conjunctivitis $[6,7]$.

The mainstay conventional treatment for AR includes allergen avoidance, pharmacotherapy, immunotherapy and patient education $[1,4]$. Although the recommended first-line therapy (nasal glucocorticosteroids and leukotriene receptor antagonists) $[1,4,8]$ may be effective in reducing the symptoms of AR, the concomitant adverse effects during long-term use make them awedness. For example, second-generation $\mathrm{H} 1$ antihistamines which are frequently used for AR have limited efficacy in treating nasal congestion, commonly induce headache and drowsiness $[9,10]$. These first-line drugs can be successfully used to control AR. However, once these medications are terminated, the majority of AR patients will reappear the symptoms of AR within a brief period. Thus, these medications do not appear to exert a long-term effect on the baseline Total Nasal Symptom Score (TNSS).

Recently, the role of probiotics in nutritional interventions have been investigated regarding to their beneficial effects on AR in improving patients' quality of life and reducing medication use [11, 12]. The beneficial roles of probiotics in allergic diseases have been investigated with increasing interest in animal models and human clinical trials [13-15], which indicate their significant influence on the gut microbiota composition and host immune system restoration [16, 17]. For example, Lactobacillus casei Shirota strain exerted effect on the immune system and allergic symptoms $[18,19]$, while Lactobacillus plantarum showed the ability to inhibit allergic cytokines and Bifidobacterium longum BB536 strain alleviated symptoms of seasonal AR [20-22]. Hence, considering the unsatisfactory results of long-term AR treatment, a better understanding of relationship between gut microbiota and AR disease, and the further potential regulation mechanism of gut microbiota for AR is necessary.

Consequently, in this study, we compared the intestinal microbiota characteristics between 31 non-AR and 33 AR patients through high-throughput 16S rRNA gene sequencing. The present study was to: (1) compare the composition and functional features of gut microbiota between AR and non-AR individuals; (2) identify the specific gut microbiota which was closely related to the severity of AR as well as the potential pathway of gut microbiota interacting with the host.

\section{Methods and materials}

\section{Clinical trial number}

The ethics committee of Xiamen hospital of Traditional Chinese Medicine approved this study (Permit Number ID: 2019-K020-01) and later it was registered in
Chinese Clinical Trial Registry (ChiCTR1900028613). All healthy individuals and patients made agreement on the informed consent, and the study protocol complied with the ethical guidelines of the Declaration of Helsinki [23].

\section{Non-AR Individuals}

Thirty one non-AR populations were enrolled from December 2017 to May 2018 at Zhongshan Hospital, affiliated with Xiamen University (Xiamen, China). The inclusion criteria were as follows: (1) no allergic history and no family history of allergic diseases; (2) sausage or snake shaped stool; and (3) voluntary participation in this study. The exclusion criteria of non-AR populations were based on previously reported [24] and individuals with allergy disease, such as AR, asthma, eczema and urticarial were further excluded.

\section{Study subjects and sample collection}

Thirty three AR patients were recruited at Xiamen hospital of Traditional Chinese Medicine (Xiamen, China) from December 2019 to March 2020. Patients were recruited with the following inclusion criteria: (1) age of 18-65 years; (2) meet the international diagnostic standard of AR (Allergic Rhinitis and its impact on Asthma, ARIA) [25]: (a) the symptoms of sneezing, nasal congestion, nasal itching and rhinorrhea appear two or more, and the symptoms last or accumulate for more than 1hour every day; (b) pale and edematous nasal mucosa, nasal water like secretion; (c) at least one allergen skin prick test (SPT) and/or serum specific IgE was positive; (3) greater than or equal to 4 days every week, and four or more consecutive weeks; and (4) voluntary participation in this study.

AR patients who meet one of the following criteria were excluded: (1) upper respiratory tract infection, chronic obstructive pulmonary disease, emphysema, bronchiectasis, tuberculosis, pneumonia, bronchial asthma, secretory otitis media; (2) postural hypotension, organic heart disease or clinically significant ECG changes; (3) patients with thyroid disease, diabetes mellitus, hypoglycemia, cardiovascular and cerebrovascular disease, prostatic hypertrophy, glaucoma, epilepsy history, liver and kidney dysfunction, hematopoietic dysfunction, gastrointestinal diseases, malignant tumors; (4) patients had a history of mental and neurological diseases; (5) patients who had received the following treatment before the experiment: immunotherapy within 12 months, long-acting glucocorticoid injection therapy within 3 months, asmidazole within 6 weeks, glucocorticoids for systemic or local use within 2 weeks (inhalation or nasal spray), sodium cromoglycerin or naidomide within 1 month, long-acting antihistamines within 2 weeks, short-acting antihistamines within 1 week, ketotifen, tricyclic antidepressants, macrolides and antifungal drugs within 2 weeks, systemic 
or local nasal decongestants within $24 \mathrm{~h}$, using anti-histamine drugs, anticholinergics and antiarrhythmic drugs currently, using barbiturates, antispasmodics, phentolamine and digitalis drugs currently; or (6) abnormal physical examination results with clinical significance before admission, which may affect the achievement of the purpose of this study or hinder the realization of compliance with the research procedures according to the judgment of the researchers.

\section{TNSS and Rhinoconjunctivitis Quality of Life Questionnaire (RQLQ)}

TNSS and RQLQ were performed for AR patients to assess the severity of AR and the impact on patients' quality of life caused by AR during the enrollment. Nasal symptoms were assessed using the TNSS score [26]. RQLQ consist of 28 items in seven domains and each dimension was scored separately, and the cumulative total score was the total RQLQ score [27, 28].

\section{Lab work and the bioinformatics analysis}

The procedure for DNA extraction of fecal samples (approximately $0.25 \mathrm{~g}$ ) from non-AR healthy individuals and AR patients, the purity and concentration of the isolated DNAs, the 16S rRNA V3-V4 hypervariable region amplification for the library construction and paired-end sequencing were based on previous procedure [24]. The bioinformatics analysis procedure for raw reads were according to the previously described $[24,29]$.

\section{Statistical analysis}

Bacterial alpha diversity indices, bacterial community ordination based on Bray-Curis distance, PERMANOVA and anosim analysis were based on the $\mathrm{R}$ package vegan (version 2.5.5) [30]. The group difference comparison were conducted by ANOVA tests. The significantly distinguished taxa and predicted pathway by PICRUSt [31] were screened by comparison between AR and non-AR individuals by Wilcox test and LEfSe analysis. The sankey plots showing the genus features for AR and non-AR group were made by networkD3 packages (version 0.4). Pearson's correlations between the abundances of differential genus taxa and pathway were computed by the $R$ package stats (version 3.6.0) and package pheatmap (version 1.0.12) were used to conduct the correlation heatmap. Networks were visualized using the Gephi 0.9.1 [32]. These analyses were performed using R3.5.1 [33].

\section{Results}

\section{The clinical characteristics of participant}

In this study, we used feces collected from 33 AR patients and 31 non-AR individuals (Additional file 1: Table S1). There was no significantly difference in age, body mass index (BMI) between AR and non-AR group (AR adults between the ages of 19 and 56 years; Non-AR between the ages of 18 and 55 years). SPT (Dust mite drops) was positive in all AR patients. Among AR individuals, the TNSS was $7.33 \pm 2.41$ (Nasal obstruction: $1.70 \pm 0.92$, rhinorrhea: $1.91 \pm 1.01$, nasal itching: $1.67 \pm 0.92$, sneezing: $2.06 \pm 0.79$.) and the RQLQ was $68.73 \pm 35.76$ (Sleep problems: $5.91 \pm 4.78$, non-eye/nasal symptoms: $16.76 \pm 10.70$, practical problems: $9.52 \pm 5.19$, eye symptoms: $6.70 \pm 5.88$, nasal symptoms: $12.15 \pm 5.72$, activity limitation: $9.70 \pm 4.66$, emotions problems: $8.00 \pm 5.77$ ) (Table.1).

\section{Comparison of bacterial diversity between $A R$ and non-AR individuals}

A total of $5,875,233$ raw reads $(2,199,830$ reads for AR patients; 3,675,403 reads for non-AR individuals), average of 91,800 OTU sequence/sample and an average 316 OTUs were obtained from 64 samples (Additional file 1: Table S2). The saturated rarefaction curves based on Chao1 and observed species indicated the high quality of sequence for all samples and the score of AR samples tended to be higher than the counterpart (Additional file 2: Figure S1). Ten iterations were made to obtain

Table 1 Clinical characteristics of AR and non-AR subjects

\begin{tabular}{lll}
\hline Group & Adults & \\
\cline { 2 - 3 } & Non-AR & AR \\
\hline Subjects (n) & 31 & 33 \\
Age (years) & $32.06 \pm 9.26$ & $31.79 \pm 9.91$ \\
Gender & & \\
Male (\%) & 18 & 11 \\
Female (\%) & 13 & 22 \\
BMI (kg/m) ${ }^{2}$ & $21.85 \pm 2.57$ & $21.86 \pm 3.16$ \\
SPT (\%) & 0 & 100 \\
TNSS & 0 & $7.33 \pm 2.41$ \\
Nasal obstruction & 0 & $1.70 \pm 0.92$ \\
Rhinorrhea & 0 & $1.91 \pm 1.01$ \\
Nasal itching & 0 & $1.67 \pm 0.92$ \\
Sneezing & 0 & $2.06 \pm 0.79$ \\
RQLQ & NA & $68.73 \pm 35.76$ \\
Sleep problems & NA & $5.91 \pm 4.78$ \\
Non-eye/nasal symptoms & NA & $16.76 \pm 10.70$ \\
Practical problems & NA & $9.52 \pm 5.19$ \\
Eye symptoms & NA & $6.70 \pm 5.88$ \\
Nasal symptoms & NA & $12.15 \pm 5.72$ \\
Activity limitation & NA & $9.70 \pm 4.66$ \\
Emotions problems & NA & $8.00 \pm 5.77$ \\
\hline
\end{tabular}

\# means $p>0.05$, no significant effect. NA: Not available. SPT, skin prick test, TNSS, Total Nasal Symptom Score. RQLQ, Rhinoconjunctivitis Quality of Life Questionnaire 
the alpha diversity metrics. There were significant differences between AR and non-AR individuals in diversity indices Chaol and Shannon $(p<0.05)$ while not for $\mathrm{J}$ indices $(p>0.05)$ (Fig. 1a). For example, the average observe, Chao 1 and ACE index from the AR patients $(330,421,418)$ were twice times more than that of non-AR individuals $(161,207,202)$ while the Simpson $(0.87)$ and $\mathrm{J}$ indices (0.57) were similar (Additional file 1: Table S3). Shared "universal" OTUs (found in samples from all AR and non-AR patients) accounted for $58.5 \%$ of the total. Non-AR individuals contained more exclusive OTUs than that of AR patients (Fig. 1b).

Significant differences in microbial community structure between AR and the non-AR group were observed based on ANOSIM analyses $(p<0.001)$ (Additional file 2: Figure S2A). From the PCoA plot that were based on the Bray-Curtis distance, the microbiota was separated in the first axis (Fig. 1c, d) and the non-AR group tended to be more dispersed than AR patients which could be further verified by distance variation analysis (Additional file 2: Figure S2B), the Bray-Curtis dissimilarity within
non-AR individuals were significantly higher than that of within AR group.

\section{Comparison of bacterial community between AR and non-AR individuals}

Sixteen bacterial phyla were detected in total, Thereinto, Bacteroidetes, Firmicutes, Proteobacteria, Fusobacteria were the dominated taxa (occupying approximately 99.0\%). In AR group, the average relative abundance of Bacteroidetes $(49.27 \%)$ and Proteobacteria $(3.61 \%)$ were lower than that in non-AR individuals $(54.32 \%, 6.25 \%$ separately) while Chloroflexi and Spirochaetes were the unique taxa which were absent in non-AR parts (Additional file 1: Tables S4 and S5). Non-AR group had lower abundance of Firmicutes and Fusobacteria, but had the unique phyla Lentisphaerae (Fig. 2a). Two hundred genus were observed in total and Bacteroides, Prevotella_9, Phascolarctobacterium, Faecalibacterium, Roseburia, Escherichia, Shigella, Romboutsia, Megamonas, Alistipes, Lachnoclostridium were the top 10 genus which added up to $75.20 \%$ (Additional file 1: Table S6). Compared with non-AR group, AR patients displayed higher abundance

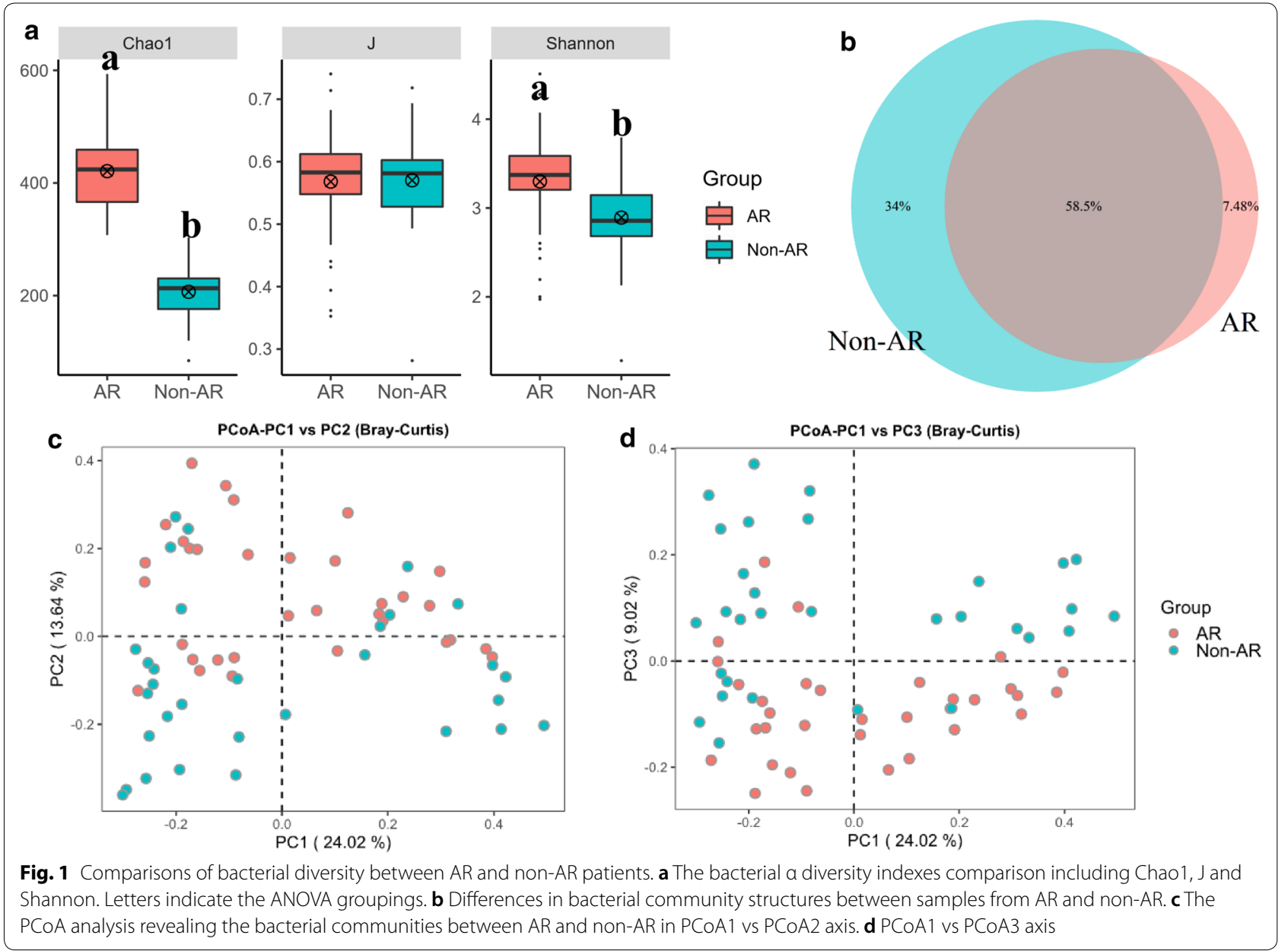




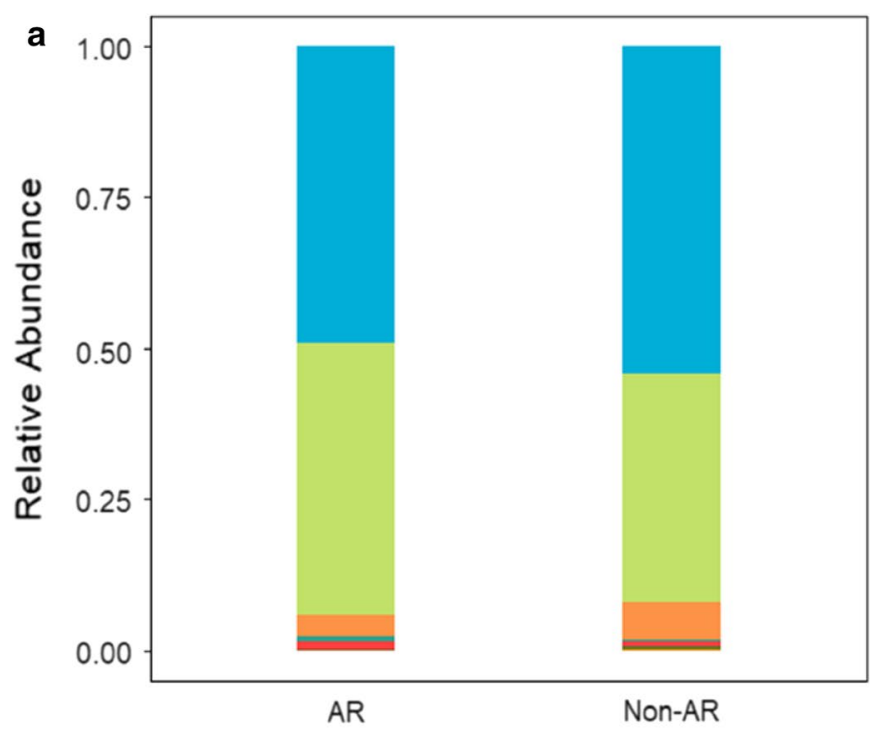

\section{Phylum}

Bacteroidetes

Firmicutes

Proteobacteria

Actinobacteria

Fusobacteria

Cyanobacteria

Patescibacteria

Euryarchaeota

Epsilonbacteraeota

Synergistetes

Verrucomicrobia

Others



Genus

Bacteroides

Prevotella_9

Phascolarctobacterium

Faecalibacterium

Roseburia

Escherichia.Shigella

Romboutsia

Megamonas

Alistipes

Lachnoclostridium

un f Lachnospiraceae

Pârābacteroides

Lachnospira

Fusobacterium

Veillonella

Ruminococcaceae_UCG.002

Subdoligranulum

Blautia

Sutterella

Others

Fig. 2 The bacterial structure comparison between AR and non-AR in phylum (a) and genus (b) level. Top 20 genus according to the relative abundance were included

of Prevotella_9, Phascolarctobacterium, Faecalibacterium, Parabacteroides while lesser in Bacteroides, Megamonas, Romboutsia and Veillonella (Fig. 2b and 3).

Furthermore, the wilcox test were made to compare the significantly distinguished phyla and genus between AR and non-AR group. Firmicutes, Fusobacteria, Actinobacteria, Cyanobacteria and Chloroflexi were the five differential phyla (Fig. 4a). There were 76 significantly different genus and Prevotella_9, Phascolarctobacterium, Roseburia, Megamonas, Alistipes and Lachnoclostridium were the top ranked distinguished genus (Table2, Additional file 1: Table S7, Fig. 4b).

\section{Higher complicated network pattern in AR patients}

For the non-AR group, whose modularity, nodes and edges were 0.86, 142 and 45 separately, Actinobacteria, Bacteroidetes and Proteobacteria might play more important roles than that of in AR, and Slackia, Raoultibacter that are within Actinobacteria, were highly correlated with Eubacterium brachy, DTU089 within Firmicutes. In the AR group, the clustered nodes mainly included Anaerofilum, Olsenella, Pseudobutyrivibrio within Firmicutes, Olsenella within Actinobacteria (Additional file 1: Table S8). The edges, nodes, modularity for AR group were 125,142 and 0.67 


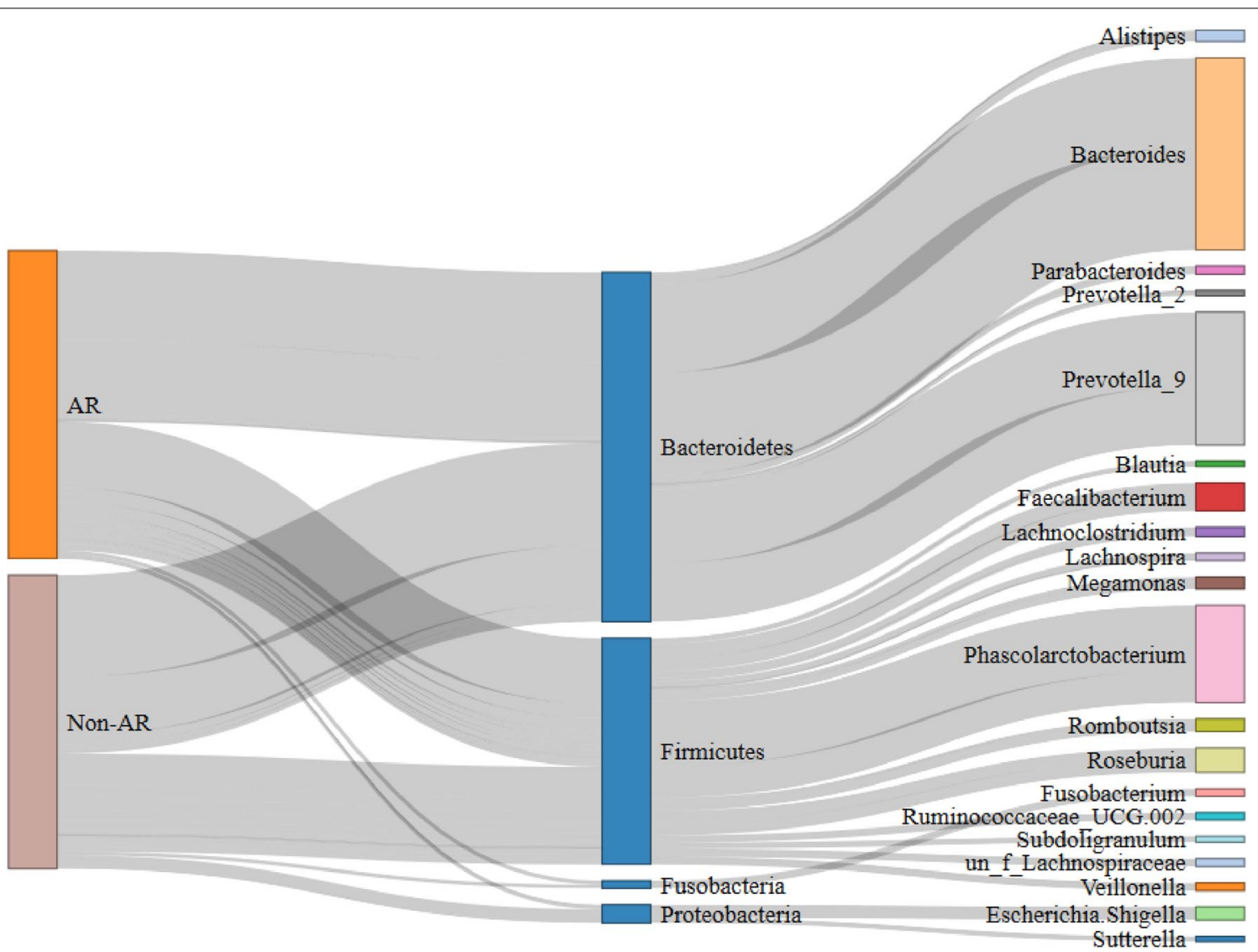

Fig. 3 Sankey analysis of all non-AR and AR patients
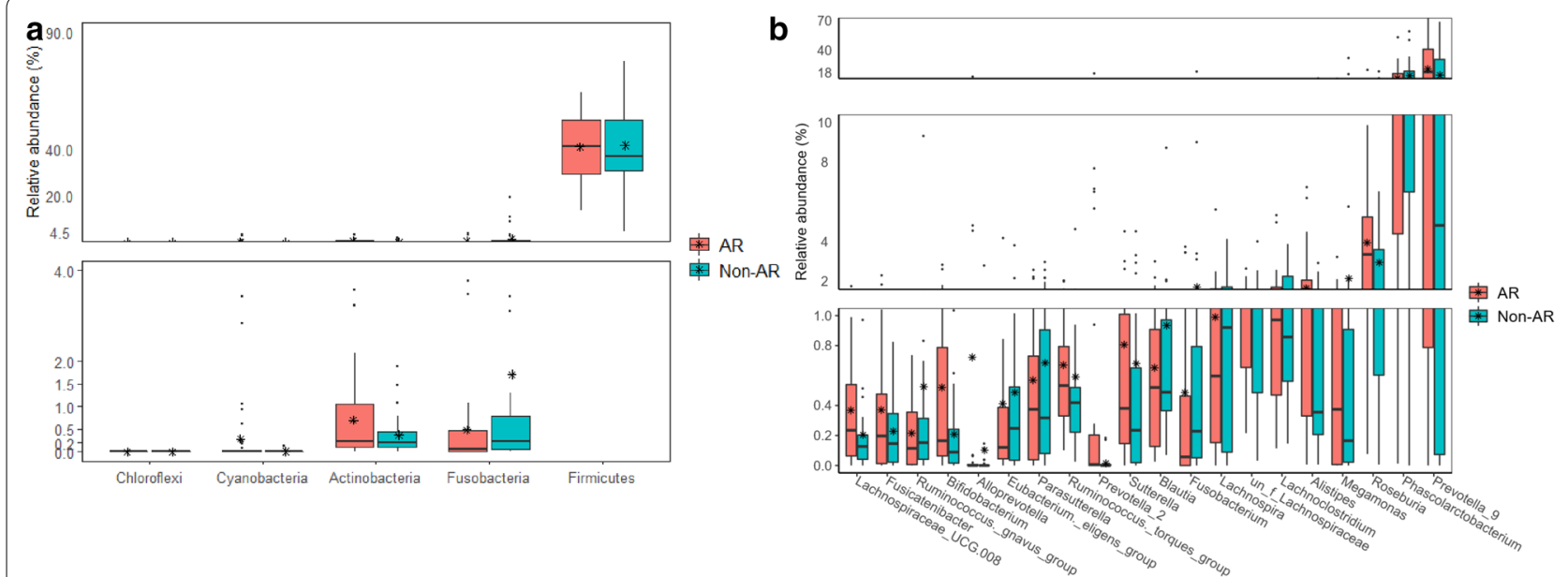

Fig. 4 The relative abundance of distinguished bacterial taxa between non-AR and AR patients. a Differences in relative abundances of distinguished phyla screened by wilcox test. $\mathbf{b}$ Differences in relative abundances of top 20 distinguished genus screened by wilcox test $(p<0.05)$ and $|\log \mathrm{FC}|>1$ 
Table 2 Top 20 distinguished genus between AR patients and non-AR healthy individuals

\begin{tabular}{lcr}
\hline Genus & AR(Average(Min-Max)) & Non-AR(Average(Min-Max)) \\
\hline Prevotella_9 & $0.211(0.004-0.779)$ & $0.163(0-0.548)$ \\
Phascolarctobacterium & $0.185(0.038-0.593)$ & $0.088(0-0.304)$ \\
Roseburia & $0.035(0.009-0.126)$ & $0.034(0-0.209)$ \\
Megamonas & $0.012(0-0.120)$ & $0.022(0-0.328)$ \\
Alistipes & $0.006(0.001-0.053)$ & $0.026(0-0.123)$ \\
Lachnoclostridium & $0.019(0.004-0.053)$ & $0.008(0.001-0.029)$ \\
un_f_Lachnospiraceae & $0.013(0.004-0.026)$ & $0.009(0-0.040)$ \\
Lachnospira & $0.019(0.006-0.056)$ & $0.003(0-0.013)$ \\
Fusobacterium & $0.011(0-0.193)$ & $0.010(0-0.107)$ \\
Blautia & $0.009(0.002-0.022)$ & $0.007(0-0.087)$ \\
Sutterella & $0.009(0-0.045)$ & $0.006(0-0.030)$ \\
Prevotella_2 & $0.003(0-0.067)$ & $0.012(0-0.174)$ \\
Ruminococcus._torques_group & $0.004(0.001-0.016)$ & $0.008(0-0.046)$ \\
Parasutterella & $0.009(0.001-0.030)$ & $0.003(0-0.015)$ \\
Eubacterium._eligens_group & $0.007(0.001-0.042)$ & $0.001(0-0.022)$ \\
Alloprevotella & $0.001(0-0.028)$ & $0.008(0-0.142)$ \\
Bifidobacterium & $0.005(0.001-0.028)$ & $0.002(0-0.018)$ \\
Ruminococcus.gnavus_group & $0.003(0-0.016)$ & $0.004(0-0.093)$ \\
Fusicatenibacter & $0.005(0.001-0.023)$ & $0(0-0.002)$ \\
Lachnospiraceae_UcG.008 & $0.004(0-0.018)$ & $0.002(0-0.010)$ \\
\hline
\end{tabular}

These 20 genus are screened out based on wilcox.test $(p<0.05)$ and $\mid \log ($ Fold change) $\mid>1$ between the two groups

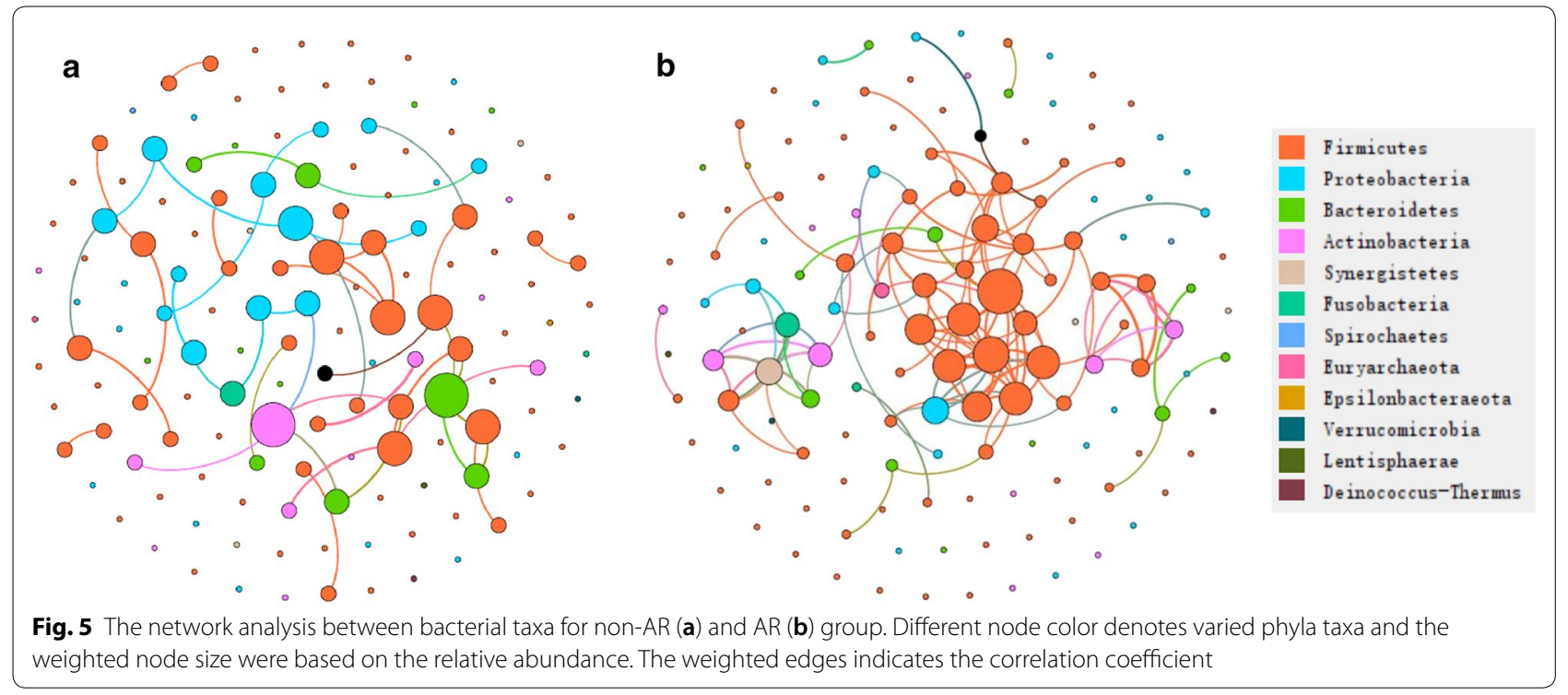

separately. Hence, AR tended to have more complicated network topology (Fig. 5).

\section{Association between featured bacterial taxa and pathway} The functional pathway based on 16S rRNA gene were predicted by Picrust to obtain the pathway or metabolites information that may be involved with AR condition.
The mean nearest sequenced taxon index (NSTI) value was $0.08 \pm 0.02$ for all samples (Additional file 1: Table S9). There were 328 detected pathway or metabolites in KEGG level 3 and the top list included transporters, ABC-transporters, purine metabolism, peptidases, pyrimidine metabolism and so on. Then the wilcox test were made to compare the significantly distinguished 
features and seven of them that were featured with $|\operatorname{logFC}|>1$ (the expression relative abundance in non-AR group or AR were twice time than AR or non-AR) were screened out (Additional file 1: Table S10). The results showed that the pathway associated with alpha linolenic acid metabolism and bacterial invasion of epithelial cells were significant higher in non-AR group as compare with that of $A R$, while AR patients showed the higher expression of GnRH signaling pathway, Fc $\gamma$-R mediated phagocytosis and Endocytosis (Additional file 2: Figure S3). Furthermore, the correlationship between seven distinguished pathway and the top 20 significantly differed genus were calculated to reveal the potential interaction mode. As is depicted in Fig. 6, no genus significantly related with Shigellosis which were caused by bacterium genus Shigella. Prevotella_9 negatively related with alpha linolenic acid metabolism. Both Eubacterium eligens and Parasutterella were negatively correlated with Endocytosis, GnRH signaling pathway and Fc $\gamma$-R mediated phagocytosis.

\section{Moderate and severe AR patients had similar bacterial communities}

Based on the TNSS score and actual small sample size, we divided 33 AR patients into moderate $(0-7)$ and severe (8-12) group to compare the bacterial diversity and community to probe into the role of bacterium in the progression of AR. Although moderate and severe AR patients showed significant difference in non-eye/ nasal symptoms, practical problem, nasal symptom, eye symptoms, emotions and RQLQ (Additional file 1: Table S11), the $\alpha$ and $\beta$ diversity displayed no significant difference (Additional file 2: Figure S4), which indicated that at least in this research, bacterial taxa played less roles in the AR progression.

The TNSS score is calculated based on the sum up of nasal obstruction, rhinorrhea, nasal itching, and sneezing, which represents perspectives of AR symptom, that is, the higher score means the worse symptom condition. RQLQ score which consists of activity limitation, sleep disorders, non-eye/nasal symptoms, practical problem, nasal symptom, eye symptoms and emotions, reflects the life quality status that associated with $\mathrm{AR}$ disease. The value for each score and RQLQ positively correlated with the extent of the AR impact on the life quality. Here the spearman rank correlation analysis were made to associate TNSS and RQLQ score with bacterial genus. Butyricicoccus, Mailhellam and Marvinbryantia were significantly positive-correlated with TNSS score while opposite for Filifactor. Butyricicoccus, Lachnospiraceae_UCG.001, Marvinbryantia, Prevotella_2, Ruminococcaceae_UCG.013, Slackia were the significant genus that positively related with RQLQ while Eisenbergiella were negatively associated with RQLQ, eye symptoms, nasal symptom, practical problem, non-eye/ nasal symptoms which might need further verification for the therapeutic target of AR disease (Additional file 1: Table S12).
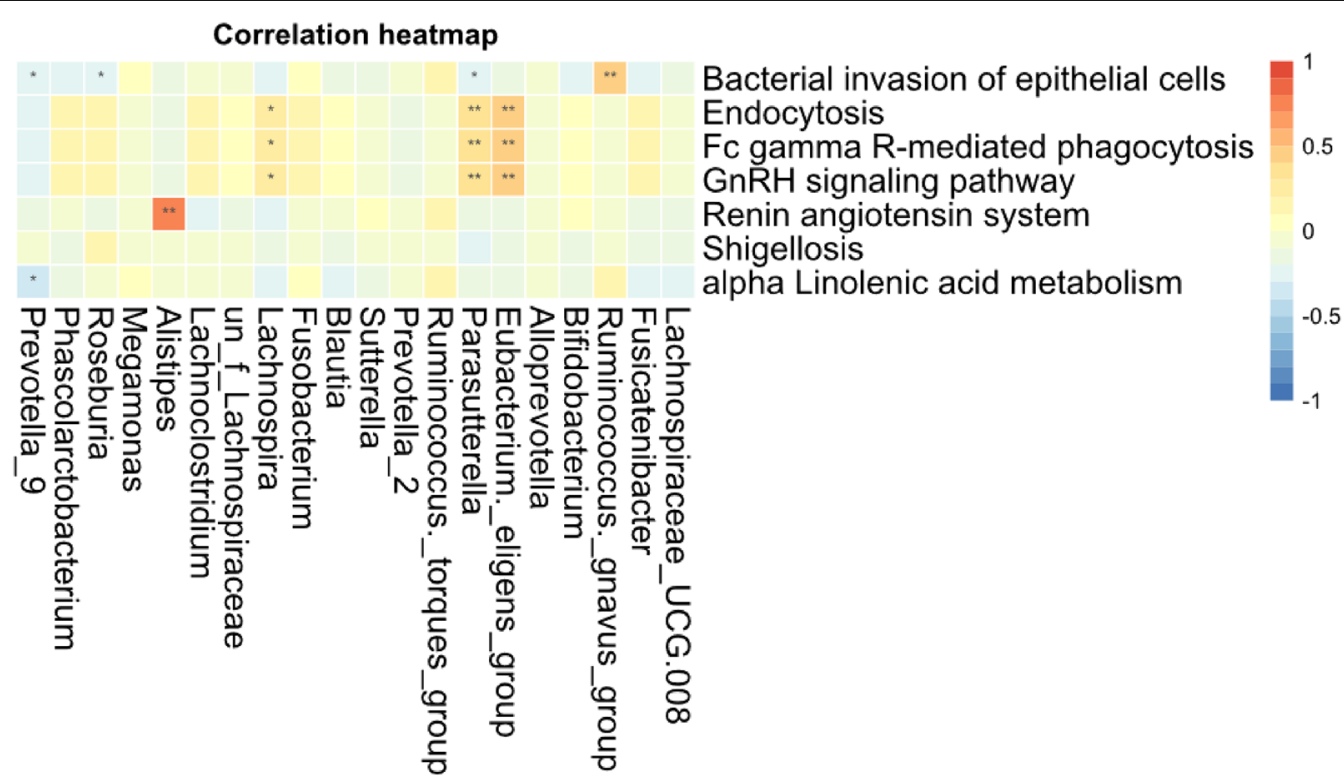

Fig. 6 The correlation heatmap between distinguished predicted pathway and bacterial genus. Both differential genus and predicted pathway were screened based on wilcox test $(p<0.05)$ and $|\log F C|>1$. Correlation significance, ${ }^{*}$ denotes $p<0.05$ and ${ }^{* *} p<0.01$ 


\section{Discussion}

Human gut microbes are key players in host immune responses, metabolism and allergy disease progression [34]. During the study, we compared the composition and functional profiles of gut microbiota between AR patients and their counterparts. The bacterial richness and diversity in AR group was significantly higher than that of non-AR group, which was different from previous findings [35-37]. Most previous studies have shown that the microbial diversity of AR decreased as comparing with non-AR populations [35-37] while few studies found that there was no significant difference in bacterial diversity between AR and non-AR group [38]. According to the sankey diagram of gut microbiota in this study, we found that the higher microbial diversity in AR patients might be due to the expansion of bacteria taxa within Firmicutes.

Generally, opportunistically pathogenic bacteria within healthy gut exerted no problems in immunecompetent hosts while outgrowth of these organisms can contribute to disease [37]. For example, larger proportions of Firmicutes have been reported to be associated with atopic disease, especially in the development of AR and asthma in early childhood [39, 40]. More complicated network topology formed by the expansion of bacterial taxa within Firmicutes in AR group was observed in this study, which indicated the stubborn and leading roles of them in the cross-talk of bacterial community. Hence, adjusting the ecosystem network by modulating the related taxa might be the target for intervention. The proportions of the beneficial microbial organisms were lower in AR group as compared with non-AR group, such as Bacteroidetes and Proteobacteria, which have been reported to increase the total numbers of Tregs within the colon. And B. caccae, $B$. thetaiotaomicron, and $B$. vulgaris proved to play important roles in protecting host from allergy disease [37]. Also, alterations toward dysbiosis in the composition and predicted function of the gut microbiota in individuals with AR were associated with high total IgE levels [38].

Although the diversity and gut microbiota community between moderate and severe AR group displayed no significant difference, it cannot be absolutely assumed that the gut microbiota plays less role in the AR progression. Many confounding factors can cause disruptions in the structure of the microbial community such as the effect of delivery mode, birth order and family size, antibiotic exposure, diet, breast feeding, indoor or outdoor pollution, occupation, habitation, and socioeconomic factors, etc. [41, 42]. Almost all of the AR individuals included in this study were delivered naturally and breast-fed (Only 2 of 33 cases were caesarean section and 1 of 33 cases was formula fed.). Additionally, individuals in AR group have the similar birth order and family size because of China's one-child policy. Both AR and non-AR individuals live in Xiamen all year round. The lack of differences in gut microbiota between the moderate and severe AR group may be caused by the similar demographic characteristic of AR individuals in this study. Previous studies showed that the maternal vagina and breast milk consisted of Lactobacilli and Streptococci can reduce the risk of allergy disease as compare with cesarean section and formula fed [43, 44]. Hence, further studies with comparing the impact of confounding factors on AR are needed.

Although there were four primary AR symptoms including nasal congestion, nasal itching, rhinorrhea and sneezing, we found that the clinical manifestations of AR patients varied greatly in real outpatient practice. For example, some AR individuals have serious nasal itching, while other three symptoms were mild; Some AR patients have runny nose, sneezing, but no nasal itching or very mild; Some patients may only have nasal congestion, while other three symptoms were not obvious. We found that the difference of gut microbiota may pose a potential reason for these phenomena through the spearman rank correlation analysis. In this study, Candidatus_Stoquefichus was positively related with runny nose; Catenibacterium and Intestinimonas were positively related with nasal congestion, while opposite for Alloprevotella; Hydrogenoanaerobacterium and Prevotella_6 were positively related with sneezing, while Granulicatella and Porphyromonas were negatively related with sneezing; Butyricicoccus, CAG.56, Family_XIII_UCG.001, Holdemanella, and Hydrogenoanaerobacterium were positively related with nasal itching, while opposite for Eisenbergiella and Megasphaera. The above-mentioned results suggest that gut microbiota is positively involved in with the clinical manifestations in varied AR patients.

Regarding to RQLQ, Butyricicoccus, Lachnospiraceae_UCG.001, Marvinbryantia, Prevotella_2, and Ruminiclostridium were found to be positively related with activity limitation caused by AR; Butyricicoccus and Slackia were positively related with non-eye/nasal symptoms, while opposite for Eisenbergiella; Butyricicoccus, Lachnospiraceae_UCG.001, and Prevotella_2 were positively related with eye symptoms, while opposite for Eisenbergiella; Butyricicoccus, Lachnospiraceae_UCG.001, and Prevotella_2 were positively related with emotion problem;Lachnospiraceae UCG.001, Marvinbryantia, Prevotella_2, Ruminiclostridium were positively related with practical problem, while opposite for Eisenbergiella; Marvinbryantia and Prevotella_2 were positively related with nasal symptom, while opposite for Eisenbergiella; Slackia was positively related with sleep disorders. Specific probiotics may have huge 
potential value in the future, which was worthy to be developed to cope with the characteristic symptoms of AR individuals based on these findings.

The potential roles of gut microbiota in AR were predicted in this study. The expression level of predicted function associated with alpha linolenic acid metabolism and bacterial invasion of epithelial cells were higher in non-AR individuals than in AR patients. The bacterial invasion of epithelial cells pathway can destroy the mucosal barrier function which lead to the intrusion of exogenous pathogens. It was well known that the gut microbiota played a key role in the formation of mucosal immune system. In terms of function, the gut mucosa and nasal mucosa may seem as a single organ, which sharing the functions of immune surveillance as well as shaping of host responses $[45,46]$. The balance between $\mathrm{T}$ type 17 cells and $\mathrm{T}$ regulatory cells in the lamina propria of the small intestine can be regulated by the gut microbiota [45]. The symbiotic relationship between intestinal mucosa and the gut microbiome has a barrier mechanism to exclude most bacteria, so as to prevent undesired invasion and the tolerance will be developed through continuous exposure to bacterial products. In AR patients, pathobionts accounted for a large proportion which can invasion the intestinal mucosa epithelial cells leading to barrier function dysbiosis. The dendritic cells and macrophages can be affected by the pathobionts and metabolic by-products directly or through the intermediacy of epithelial cells, which is regulated by microbiota-driven epigenetic mechanisms. Besides, the microbial metabolic products also can induce the maturation of $\mathrm{T}$ regulatory cells as well as A and B cell, causing such cells to switch their immunoglobulin isotypes. For example, IgE modifies the gut microbiota via activating basophils and mast cells. The development of gut mucosal immunity which can prevent the intrusion of exogenous pathogens is stimulated by the cross-talk between the gut microbiota and the immune system [42].

There were several limitations to this study. Firstly, we included limited number of subjects in each group with a cross-sectional design confined to Xiamen area, which remind of us that more samples across different geographic area should be considered to make more robust conclusion. Secondly, although we know diet, lift style and many other confounding factors induce gut microbiota dysbiosis, the effect of environmental factors, including indoor, outdoor pollution, occupation, habitation and socioeconomic factors were not included in the analysis [47]. Thirdly, biomarkers of respiratory inflammation such as peripheral blood eosinophil, nasal mucus eosinophil or IgE were not fully assessed in this study. Fourth, although 16S rRNA PICRUSt analysis has been adopted in this human gut microbiota research to help us further comprehend the potential function and pathway related with allergy rhinitis, the less accurate results associated the inherent drawback of database and tools should be further improved by metagenome, transcriptome and other omics tools. In addition, although we collected the nasal mucosa flora, the quantity of the nasal mucosa flora was too small to carry out $16 \mathrm{~S}$ rRNA sequencing that we could not know the potential influence of upper airway microbiota on AR. AR seems to be a self-healing disease with the growing of age and increasing of immunity for some AR children. But clinically, we still see a lot of AR patients, who suffering from AR from children to adults and even to the elderly. Unfortunately, this study did not include the children or the elderly AR patients, so we do not know which certain microbiota plays a key role in the progress of AR. Hence, further studies of larger sample size, multi-omics, host-microbiome interaction are needed to validate these findings, which will guide us the precise roles of gut microbiota in the pathogenesis and progression of AR.

\section{Conclusion}

Both the bacterial $\alpha$ and $\beta$ diversity were significantly different between AR and non-AR individuals. The potentially beneficial bacterial related to reduce nasal symptoms and improve the patients' quality of life were lower in AR patients, whereas taxa associated with enhancement of nasal symptoms and decrease of the patients' quality of life were higher in AR patients. These results suggest that the alteration of gut microbiota can be associated with AR through their functional roles. This study sets the basis for gut microbiota regulation as a potential therapeutic target for allergy rhinitis.

\section{Supplementary information}

Supplementary information accompanies this paper at https://doi. org/10.1186/s12934-020-01430-0.

Additional file 1: Table S1. Basic clinical information of included patients and healthy individuals. Table S2. Sequence process information about AR patients and non-AR healthy individuals. Table S3. Bacterial diversity among AR patients and non-AR healthy individuals. Table S4. Bacterial taxa abundance in phyla level. Table S5. Characteristic bacterial biomarkers screened by LEfSe. Table S6. Bacterial taxa abundance in genus level. Table S7. Top 20 distinguished genus between AR patients and non-AR healthy individuals. Table S8. The nodes and edge information of non-AR healthy and AR group. Table S9. The NSTI values in each sample indicate the accuracy of the predicted metabolic potential. Table S10. The distinguished predicted pathway between AR patients and non-AR healthy individuals. Table S11. Baseline comparison between mild-to-moderate and severe AR patients. Table S12. The Spearman rank correlation between bacterial genus, TNSS and quality of life score.

Additional file 2: Figure S1. The rarefaction curves of all sample. (A) Observed species and (B) chao1. Figure S2. The bacterial community difference between AR and non-AR group. (A) The ANOSIM analysis are made to compare the intergroup and within group of samples. (B) The bacterial community variation comparison in $A R$ and non-AR group. 
Figure S3. The predicted expression of distinguished pathway. The significantly distinguished pathway predicted by PICRUSt were screened by comparison between AR and non-AR individuals by Wilcox test and based on $|\log F C|>1$. FC denotes fold change. Figure S4. Comparisons of bacterial diversity between moderate and severe AR patients. (A) The bacterial a diversity indexes comparison between moderate and severe AR, including Chao 1, J and Shannon. Letters indicate the ANOVA groupings. (B) Differences in bacterial community structures between moderate and severe AR patients.

\section{Acknowledgements}

This work was financially supported by the National Natural Science Foundation of China (81900541) and Natural Science Foundation of Fujian province (2019J05005). We appreciate all patients and healthy individuals for participating in the research.

\section{Authors' contributions}

Conceived and designed the study: LBZ, ZRC and HZX; Participated in investigation: BY, LT, YMY and YLD; Performed formal analysis: LBZ and ZRC; Collected the resources: $L B Z, F X, W R W, B Y, L T, Y M Y, Y L D$ and ZRC; Curated the data: LBZ, ZRC, FX and HZX; Wrote the manuscript: LBZ and ZRC; Supervised the study: $L B Z, Z R C$ and HZX. All authors approved the final version of the manuscript and agreed for publication once accepted. All authors read and approved the final manuscript.

\section{Availability of data and materials}

The dataset supporting the conclusions of this article is available in the NCBI Sequence Read Archive repository under the accession number PRJNA624106 (https://submit.ncbi.nlm.nih.gov/subs/). Code and scripts used in the analyses are available upon request.

\section{Competing interests}

The authors declare that they have no competing interests.

\section{Author details}

${ }^{1}$ Department of Traditional Chinese Medicine, School of Medicine, Xiamen University, Xiamen, China. ${ }^{2}$ Internal Medicine Department of Traditional Chinese Medicine, Xiamen Hospital of Traditional Chinese Medicine, Xiamen, China. ${ }^{3}$ Department of Otorhinolaryngology, Xiamen Hospital of Traditional Chinese Medicine, Xiamen, China. ${ }^{4}$ Department of Pediatrics, Xiamen Hospital of Traditional Chinese Medicine, Xiamen, China. ${ }^{5}$ Department of Acupuncture and Tuina, Fujian University of Traditional Chinese Medicine, Fuzhou, China. ${ }^{6}$ Department of Digestive Diseases, School of Medicine, Xiamen University, Xiamen, China. ${ }^{7}$ Department of Gastroenterology, Zhongshan Hospital, Xiamen University, Xiamen, China. ${ }^{8}$ Institute for Microbial Ecology, School of Medicine, Xiamen University, Xiamen, China.

\section{Received: 26 May 2020 Accepted: 25 Auqust 2020}

Published online: 01 September 2020

\section{References}

1. Bousquet J, Khaltaev N, Cruz AA, Denburg J, Fokkens WJ, Togias A, Zuberbier T, Baenacagnani CE, Canonica GW, Van WC. Allergic rhinitis and its impact on asthma (ARIA) 2008 update (in collaboration with the World Health Organization, GA(2)LEN and AllerGen). Allergy. 2008;63(s86):8-160.

2. Han MW, Kim SH, Oh I, Kim YH, Lee J. Serum IL-1 beta can be a biomarker in children with severe persistent allergic rhinitis. Allergy Asthma Clin Immunol. 2019;15:58.

3. Ruby P. Allergic rhinitis and its impacts on asthma: an evidencebased treatment strategy for allergic rhinitis. J Allergy Clin Immunol. 2001;108(5):S147-S334.

4. Seidman MD, Gurgel RK, Lin SY, Schwartz SR, Baroody FM, Bonner JR, Dawson DE, Dykewicz MS, Hackell JM, Han JK, Ishman SL, Krouse HJ, Malekzadeh S, Mims JW, Omole FS, Reddy WD, Wallace DV, Walsh SA, Warren BE, Wilson MN, Nnacheta LC. Clinical practice guideline: allergic rhinitis. Otolaryngol Head Neck Surg. 2015;152(1 Suppl):S1-S43.
5. Okubo K, Kurono Y, Ichimura K, Enomoto T, Okamoto Y, Kawauchi H, Suzaki H, Fujieda S, Masuyama K. Japanese guidelines for allergic rhinitis 2017. Allergol Int. 2017;66(2):205-19.

6. De Bot CM, Roder E, Pols DH, Bindels PJ, van Wijk RG, van der Wouden $J C$, Moed H. Sensitisation patterns and association with age, gender, and clinical symptoms in children with allergic rhinitis in primary care: a crosssectional study. Prim Care Respir J. 2013;22(2):155-60.

7. Kim MH, Hong S, Kim H, Seo H, Kim K, Ko S, Choi I. A multicenter study on the efficacy and safety of So-Cheong-Ryong-Tang for perennial allergic rhinitis. Compl Ther Med. 2019;45:50-6.

8. Brozek JL, Bousquet J, Agache I, Agarwal A, Bachert C, Bosnic-Anticevich S, Brignardello-Petersen R, Canonica GW, Casale T, Chavannes NH, Correia DSJ, Cruz AA, Cuello-Garcia CA, Demoly P, Dykewicz M, Etxeandia-Ikobaltzeta I, Florez ID, Fokkens W, Fonseca J, Hellings PW, Klimek L, Kowalski S, Kuna P, Laisaar KT, Larenas-Linnemann DE, Lodrup CK, Manning PJ, Meltzer E, Mullol J, Muraro A, O'Hehir R, Ohta K, Panzner P, Papadopoulos N, Park HS, Passalacqua G, Pawankar R, Price D, Riva JJ, Roldan Y, Ryan D, Sadeghirad B, Samolinski B, Schmid-Grendelmeier P, Sheikh A, Togias A, Valero A, Valiulis A, Valovirta E, Ventresca M, Wallace D, Waserman S, Wickman M, Wiercioch W, Yepes-Nunez JJ, Zhang L, Zhang Y, Zidarn M, Zuberbier T, Schunemann HJ. Allergic rhinitis and its impact on asthma (ARIA) guidelines-2016 revision. J Allergy Clin Immunol. 2017;140(4):950-8.

9. Bachert C, Kuna P, Sanquer F, Ivan P, Dimitrov V, Gorina MM, van de Heyning P, Loureiro A. Comparison of the efficacy and safety of bilastine $20 \mathrm{mg}$ vs desloratadine $5 \mathrm{mg}$ in seasonal allergic rhinitis patients. Allergy. 2009;64(1):158-65.

10. Kuna P, Bachert C, Nowacki Z, van Cauwenberge P, Agache I, Fouquert L, Roger A, Sologuren A, Valiente R. Efficacy and safety of bilastine 20 mg compared with cetirizine $10 \mathrm{mg}$ and placebo for the symptomatic treatment of seasonal allergic rhinitis: a randomized, double-blind, parallel-group study. Clin Exp Allergy. 2009;39(9):1338-477.

11. Zajac AE, Adams AS, Turner JH. A systematic review and meta-analysis of probiotics for the treatment of allergic rhinitis. Int Forum Allergy Rhinol. 2015;5(6):524-32.

12. Das RR, Singh M, Shafiq N. Probiotics in treatment of allergic rhinitis. World Allergy Organ J. 2010;3(9):239-44.

13. Boyle RJ, Tang ML. The role of probiotics in the management of allergic disease. Clin Exp Allergy. 2006;36(5):568-76.

14. Kalliomaki M, Salminen S, Arvilommi H, Kero P, Koskinen P, Isolauri E. Probiotics in primary prevention of atopic disease: a randomised placebo-controlled trial. Lancet. 2001;357(9262):1076-9.

15. Ouwehand AC. Antiallergic effects of probiotics. J Nutr. 2007;137(3 Suppl 2):794S-7S.

16. Eckburg PB, Bik EM, Bernstein CN, Purdom E, Dethlefsen L, Sargent M, Gill SR, Nelson KE, Relman DA. Diversity of the human intestinal microbial flora. Science. 2005;308(5728):1635-8.

17. Kalliomaki M, Isolauri E. Pandemic of atopic diseases-a lack of microbial exposure in early infancy? Curr Drug Targets Infect Disord. 2002;2(3):193-9.

18. Ivory K, Chambers SJ, Pin C, Prieto E, Arques JL, Nicoletti C. Oral delivery of Lactobacillus casei Shirota modifies allergen-induced immune responses in allergic rhinitis. Clin Exp Allergy. 2008;38(8):1282-9.

19. Tamura M, Shikina T, Morihana T, Hayama M, Kajimoto O, Sakamoto A, Kajimoto Y, Watanabe O, Nonaka C, Shida K, Nanno M. Effects of probiotics on allergic rhinitis induced by Japanese cedar pollen: randomized double-blind, placebo-controlled clinical trial. Int Arch Allergy Immunol. 2007;143(1):75-82.

20. Xiao JZ, Kondo S, Yanagisawa N, Takahashi N, Odamaki T, Iwabuchi N, Miyaji K, Iwatsuki K, Togashi H, Enomoto K, Enomoto T. Probiotics in the treatment of Japanese cedar pollinosis: a double-blind placebocontrolled trial. Clin Exp Allergy. 2006;36(11):1425-35.

21. Xiao JZ, Kondo S, Yanagisawa N, Takahashi N, Odamaki T, Iwabuchi N, Iwatsuki K, Kokubo S, Togashi H, Enomoto K, Enomoto T. Effect of probiotic Bifidobacterium longum BB536 [corrected] in relieving clinical symptoms and modulating plasma cytokine levels of Japanese cedar pollinosis during the pollen season. A randomized double-blind, placebo-controlled trial. J Investig Allergol Clin Immunol. 2006;16(2):86-93. 
22. Snel J, Vissers YM, Smit BA, Jongen JM, van der Meulen ET, Zwijsen R, Ruinemans-Koerts J, Jansen AP, Kleerebezem M, Savelkoul HF. Strainspecific immunomodulatory effects of Lactobacillus plantarum strains on birch-pollen-allergic subjects out of season. Clin Exp Allergy. 2011;41(2):232-42.

23. World MA. World Medical Association Declaration of Helsinki: ethical principles for medical research involving human subjects. JAMA 2013;310(20):2191-4.

24. Chen Z, Xie Y, Zhou F, Zhang B, Wu J, Yang L, Xu S, Stedtfeld R, Chen Q, Liu J, Zhang X, Xu H, Ren J. Featured Gut microbiomes associated with the progression of chronic hepatitis B disease. Front Microbiol. 2020;11:383.

25. Pawankar R, Bunnag C, Khaltaev N, Bousquet J. Allergic rhinitis and its impact on asthma in Asia Pacific and the ARIA update 2008. World Allergy Organ J. 2012;5:S212-S217217.

26. Aksoy C, Elsurer C, Artac H, Bozkurt MK. Evaluation of olfactory function in children with seasonal allergic rhinitis and its correlation with acoustic rhinometry. Int J Pediatr Otorhinolaryngol. 2018;113:188-91.

27. Juniper EF, Guyatt GH, Griffith LE, Ferrie PJ. Interpretation of rhinoconjunctivitis quality of life questionnaire data. J Allergy Clin Immunol. 1996;98(4):843-5.

28. Husain S, Ooi HL, Fathil N, Aziz FAA, Fuad MAM. Influence of breastfeeding on quality of life and serum IgE level in allergic rhinitis children. Bangladesh J Med Sci. 2017;16(4):530.

29. Zhang B, Xu S, Xu W, Chen Q, Chen Z, Yan C, Fan Y, Zhang H, Liu Q, Yang J, Yang J, Xiao C, Xu H, Ren J. Leveraging fecal bacterial survey data to predict colorectal tumors. Front Genet. 2019;10:447.

30. Oksanen J, Blanchet FG, Friendly M, Kindt R, Legendre P, McGlinn D, Minchin P, O Hara RB, Simpson G, Solymos P, et al. Vegan: community ecology package; 2015. https://github.com/vegandevs/vegan. Accessed 3 Jan 2020.

31. Langille MG, Zaneveld J, Caporaso JG, McDonald D, Knights D, Reyes JA, Clemente JC, Burkepile DE, Vega TR, Knight R, Beiko RG, Huttenhower C. Predictive functional profiling of microbial communities using 165 rRNA marker gene sequences. Nat Biotechnol. 2013;31(9):814-21.

32. Cherven K. Mastering Gephi Network Visualization. Birmingham: Packt Publishing Ltd; 2015.

33. R Core Team. R: a language and environment for statistical computing. $R$ Foundation for Statistical Computing. Vienna: R Core Team; 2014.

34. Blaser M, Bork P, Fraser C, Knight R, Wang J. The microbiome explored: recent insights and future challenges. Nat Rev Microbiol. 2013;11(3):213-7.

35. Abrahamsson $T$, Jakobsson $H$, Andersson A, Bjorksten $B$, Engstrand $L$, Jenmalm M. Low gut microbiota diversity in early infancy precedes asthma at school age. Clin Exp Allergy. 2014;44(6):842-50.
36. Bisgaard H, Li N, Bonnelykke K, Chawes B, Skov T, Paludan-Muller G, Stokholm J, Smith B, Krogfelt K. Reduced diversity of the intestinal microbiota during infancy is associated with increased risk of allergic disease at school age. J Allergy Clin Immunol. 2011;128(3):646-52.

37. Petersen C, Round J. Defining dysbiosis and its influence on host immunity and disease. Cell Microbiol. 2014;16(7):1024-33.

38. Chiu CY, Chan YL, Tsai MH, Wang CJ, Chiang MH, Chiu CC. Gut microbial dysbiosis is associated with allergen-specific IgE responses in young children with airway allergies. World Allergy Organ J. 2019;12(3):100021.

39. Azad MB, Konya T, Maughan H, Guttman DS, Field CJ, Chari RS, Sears MR, Becker AB, Scott JA, Kozyrskyj AL. Gut microbiota of healthy Canadian infants: profiles by mode of delivery and infant diet at 4 months. CMAJ. 2013;185(5):385-94.

40. Penders J, Stobberingh EE, van den Brandt PA, Thijs C. The role of the intestinal microbiota in the development of atopic disorders. Allergy. 2007:62(11):1223-366.

41. Prince BT, Mandel MJ, Nadeau K, Singh AM. Gut Microbiome and the development of food allergy and allergic disease. Pediatr Clin North Am. 2015;62(6):1479-92

42. Ipci K, Altintoprak N, Muluk NB, Senturk M, Cingi C. The possible mechanisms of the human microbiome in allergic diseases. Eur Arch Otorhinolaryngol. 2017;274(2):617-26.

43. Vael C, Desager K. The importance of the development of the intestinal microbiota in infancy. Curr Opin Pediatr. 2009;21(6):794-800.

44. Bieren JE, Harris NL. Microbiome and allergy. In: Encyclopedia of Immunobiology; 2016, p. 336-45.

45. Ivanov II, Frutos RL, Manel N, Yoshinaga K, Rifkin DB, Sartor RB, Finlay BB, Littman DR. Specific microbiota direct the differentiation of IL-17-producing T-helper cells in the mucosa of the small intestine. Cell Host Microbe. 2008;4(4):337-49.

46. Chiba T, Seno H. Indigenous clostridium species regulate systemic immune responses by induction of colonic regulatory $T$ cells. Gastroenterology. 2011;141(3):1114-6.

47. Kim BS, Lee E, Lee MJ, Kang MJ, Yoon J, Cho HJ, Park J, Won S, Lee SY, Hong SJ. Different functional genes of upper airway microbiome associated with natural course of childhood asthma. Allergy. 2018;73(3):644-52.

\section{Publisher's Note}

Springer Nature remains neutral with regard to jurisdictional claims in published maps and institutional affiliations.
Ready to submit your research? Choose BMC and benefit from:

- fast, convenient online submission

- thorough peer review by experienced researchers in your field

- rapid publication on acceptance

- support for research data, including large and complex data types

- gold Open Access which fosters wider collaboration and increased citations

- maximum visibility for your research: over $100 \mathrm{M}$ website views per year

At BMC, research is always in progress.

Learn more biomedcentral.com/submissions 